Papers and Proceedings of the Royal Society of Tasmania, Volume 116, 1982

(ms. received 6.5 .1981$)$

\title{
THE BRYOPHYTES OF THE MT WELLINGTON RANGE, TASMANIA
}

by D.A. and A.V. Ratkowsky

CSIRO, Hobart and Sandy Bay, Tasmania

(with five tables)

ABSTRACT

RATKOWSKY, D.A. and A.V., 1982 (3I viii): The bryophytes of the Mt Wellington Range, Tasmania. Pap. Proc. R. Soc. Tasm., 116: 97-115.

https://doi.org/10.26749/rstpp.116.97 ISSN 0080-4703. C.S.I.R.O.,Hobart, Tasmania.

A census of the bryophyte flora of the Mt Wellington Range, Tasmania, is reported, the field work having been carried out between 1 May 1977 and 31 October 1980 . The survey area was similar in extent to that used by the authors in an earlier survey of the vascular plants. A total of 164 mosses and 130 liverworts was found in the survey area, representing more than $60 \%$ of the known Tasmanian bryophyte flora, including several species not previously known to occur in Tasmania. The number of moss species was greatest at middle and lower altitudes whereas the number of liverwort species was greatest at middle and higher altitudes. Reports of liverwort species in this census are accompanied by references to works containing descriptions and drawings, wherever possible, of these species. Only a few species known from past information or collections to have occurred on Mt wellington were missing in the current survey despite the devastating bushfire of 7 February 1967.

\section{INTRODUCTION}

Serious efforts were made to study the bryophyte flora of Tasmania before that of most other parts of Australia. Bastow (1886-87) published a four-part paper (with an illustrated fold--out key) on the Tasmanian mosses and within thirty years Rodway (1913-14) brought out an extensive revision. Similarly, Bastow (1888) published an illustrated account of the Tasmanian liverworts, and Rodway (1917) provided an extensive revision. Although Rodway recognized that many of his "species" were really different forms of the same species and therefore not fully deserving of specific rank, it was only relatively recently that bryologists have brought into synonymy many of these forms previously considered to be distinct. Indeed, the plasticity of many species both in their vegetative and reproductive organs is probably at least as great as in the flowering plants, and perhaps bryologists of the future will increase the list of synonyms still further. Since Rodway's time many genuine new species have been added to the bryophyte flora of Australasia (including Tasmania) due to the efforts of such workers as H.N. Dixon, G.O.K. Sainsbury, J.H. Willis, E.A. Hodgson, K.W. Allison, R. Grolle and R.M. Schuster.

\section{SURVEY AREA AND VEGETATION ZONES}

The field work for the present survey was carried out between 1 May 1977 and 31 0ctober 1980, the survey area used being the same as that employed for a census of the vascular plants of the Mt Wellington Range (Ratkowsky \& Ratkowsky 1976). Details of the vegetation zones occurring within the survey area are given in that paper and will not be repeated here, although Table 1 summarizes the main features and approximate elevations of each zone. Unlike the earlier survey where a minimum elevation of $240 \mathrm{~m}$ was employed in order to make a comparison with a previously published survey, no lower elevation limit was used in the present survey, except that bryophytes growing in the vicinity of habitation were not included.

\section{CENSUS OF THE MOSSES}

An important publication subsequent to the revision of the moss flora by Rodway (1913-14) was the eight-part commentary of Sainsbury (1953-56) on the moss species in 


\section{TABLE 1}

SUMMARY OF THE VEGETATION ZONES OF THE MT WELLINGTON RANGE

Woodlands containing pure stands of Eucalyptus coccifera, and treeless marshlands and grasslands at the same elevations.

Wodlands with mixed eucalypt stands, dominant eucalypt same elevations. Sphinx Rock, etc., dominant eucalypt E. johnstonii. of Bedfordia salicina and olearia argophyzza.

what was then Rodway's herbarium (now incorporated into the Tasmanian Herbarium).

These papers, together with the works of J.H. Willis, and others, helped form the basis for the moss flora of southern Australia of Scott and Stone (1976). Their treatment must now be the starting point for any serious revision of the moss flora of this region. The ordering of the families and genera in the present census (Table 2) follows scott and Stone (1976), and the names of species, arranged alphabetically, adhere to those given in that work, except where more recent knowledge requires modification. Footnotes, which are listed at the end of table 2, are used for clarification, to impart extra information, or to indicate unsolved problems of taxonomic interest.

Mosses are notoriously difficult to identify with certainty in the field, as the identity of many species can usually only be determined with confidence after microscopic examination. This fact makes it difficult to accurately assess the abundance of a species in a vegetation zone. Thus, unlike the census of vascular plants (Ratkowsky $\xi$ Ratkowsky 1976), no assessment of the frequency of occurrence of a species is indicated in table 2, presence in any zone being indicated by a " $x$ ". However, where a species was found only once during the survey, its occurrence is indicated by a " $r$ " to signify that it is rare.

\section{CENSUS OF THE LIVERWORTS}

Discussion of a census of Tasmanian liverworts is complicated by the absence of a modern treatment of the hepatic flora of this region, as there is as yet no comprehensive work on liverworts equivalent to that of Scott and Stone (1976) on the mosses. There are a number of large families, notably those of Lophocolaceae and Lepidoziaceae, that are badly in need of revision. The lack of an equivalent to Scott and Stone (1976) has prompted a different approach to the presentation of the census of the liverworts of Mt Wellington in table 3. Each species listed is followed by a number indicating a footnote. The footnotes appear at the end of the table and provide references to literature either citing nomenclature or providing descriptions and illustrations, wherever possible, 
TABLE 2

THE MOSSES OF THE MT WELLINGTON RANGE

\section{Sphagnaceae}

Sphragnum australe Mitt.

S. cristatum Hampe

S. faZcatuzum Besch.

S. subsecundum (1) Nees

\section{Andreaeaceae}

Andreaec rupestris (2) Hedw.

A. subulata Harv. ex Hook.

Polytrichaceae

Polytrichum alpinum Hedw.

P. cormune Hedw.

P. juniperinum Hedw.

Atrichum androgynum (C. Muel1.) Jaeg.

Pogonatum subulatum (Brid.) Brid.

Polytrichadelphus magelzanicus (Hedw.) Mitt.

Psilopilum australe (Hook.f. E Wils.) Mitt.

P. crispuZum (Hook.f. \& Wils.) Mitt.

$\begin{array}{cccccccc}1 & 2 & 3 \mathrm{a} & 3 \mathrm{~b} & 4 & 5 & 6 & 7 \\ & & \mathrm{x} & & & \mathrm{x} & & \\ \mathrm{x} & \mathrm{x} & \mathrm{x} & & & \mathrm{x} & & \\ \mathrm{x} & \mathrm{x} & & & & & & \\ \mathrm{x} & \mathrm{x} & \mathrm{x} & & & \mathrm{x} & & \\ & & & & & & & \mathrm{x} \\ \mathrm{x} & \mathrm{x} & \mathrm{x} & \mathrm{x} & & & & \mathrm{x} \\ & & & \mathrm{r} & & & & \\ \mathrm{x} & \mathrm{x} & & & & & & \\ \mathrm{x} & \mathrm{x} & \mathrm{x} & \mathrm{x} & & \mathrm{x} & \mathrm{x} & \\ \mathrm{x} & \mathrm{x} & \mathrm{x} & \mathrm{x} & \mathrm{x} & \mathrm{x} & \mathrm{x} & \mathrm{x} \\ & & & & \mathrm{x} & & \mathrm{x} & \\ & & \mathrm{x} & \mathrm{x} & \mathrm{x} & & \mathrm{x} & \\ \mathrm{x} & \mathrm{x} & \mathrm{x} & \mathrm{x} & \mathrm{x} & \mathrm{x} & \mathrm{x} & \\ & \mathrm{x} & \mathrm{x} & & & & & \\ & & & & & & & \\ & & & & & & & \mathrm{x} \\ & & & & \mathrm{x} & \mathrm{x} & \mathrm{x} & \mathrm{x} \\ & & & & \mathrm{x} & & & \\ & & \mathrm{x} & \mathrm{x} & \mathrm{x} & \mathrm{x} & \mathrm{x} & \mathrm{x} \\ & & & \mathrm{x} & \mathrm{x} & \mathrm{x} & \mathrm{x} & \mathrm{x} \\ & & & \mathrm{x} & \mathrm{x} & & \mathrm{x} & \mathrm{x} \\ & & & & \mathrm{x} & \mathrm{x} & \mathrm{x} & \mathrm{x} \\ & & & & & & \mathrm{x} & \mathrm{x} \\ & & & \mathrm{x} & \mathrm{x} & \mathrm{x} & & \mathrm{x} \\ & & & & & & & \mathrm{r} \\ & & & & & & & \mathrm{x} \\ \mathrm{x} & \mathrm{x} & \mathrm{x} & \mathrm{x} & \mathrm{x} & \mathrm{x} & \mathrm{x} & \\ \mathrm{x} & \mathrm{x} & \mathrm{x} & \mathrm{x} & \mathrm{x} & \mathrm{x} & \mathrm{x} & \mathrm{x} \\ \mathrm{x} & \mathrm{x} & \mathrm{x} & \mathrm{x} & & & & \end{array}$

Fissidens aspienioides Hedw.

$F$. dealbatus Hook.f. \& Wils.

F. Leptocladus C. Muell. \& Rodw.

$F$. pallidus Hook.f. \& Wils.

$F$. pungens C. Muel1. \& Hampe

F. rigidulus Hook.f. \& Wils.

$F$. tayzorit C. Muell.

$F$. tenellus Hook.f. \& Wils.

$F$. vittatus Hook.f. \& Wils.

Grimmiaceae

Grimmia apocarpa (Hedw.) B.S.G.

G. Laevigata (Brid.) Brid.

G. puZvinata (Hedw.) Sm.

G. trichophyzia Grev.

Rhacomitrium crispulum (Hook.f. \& Wils.) Dix

R. Zanuginosum (Hedw.) Brid. var. pruinosum Wils.

Ditrichaceae

Ditrichum cylindricarpum (C. Muell.) F. Muell.

D. difficile (Dub.) Fleisch.

D. punctulatum Mitt.

Ceratodon purpureus (Hedw.) Brid.

Eccremidium pulchellum (Hook.f. \& Wils.) C. Muell.

Cheilothela chilensis (3) (Mont.) Broth.

Seligeriaceae

Blindia magelzanica Schimp.

$B$. robusta Hampe

B. tasmanica Sainsb.

Dicranaceae

Campyzopus clavatus (R.Br.) Wils.

C. introflexus (Hedw.) Brid.

C. palzidus Hook.f. \& Wils.

Dicranella cardotii (R.Br. ter.) Dix.

D. dietrichiae (4) (C. Muell.) Jaeg.

Dicranoloma bizlardieri (Brid.) Par.

D. dicarpum (Nees) Par.

D. menziesii (Tay1.) Par.

$\mathrm{x} \quad \mathrm{x}$ 
Dicranoweisia microcarpa (Hook.f. \& Wils.) Par. Leucobryum candidum (P. Beauv.) Wils.

Pottiaceae

Acauzon integrifolium C. Mue11.

Calyptopogon mioides (Schwaegr.) Broth.

Tortula muralis Hedw.

T. papillosa Wils.

T. princeps De Not.

T. mbra (5) Mitt.

Gymostomum calcareum Nees \& Hornsch.

Tortelza calycina (Schwaegr.) Dix.

T. knightii (Mitt.) Broth.

Tridontium tasmanieum Hook. f.

Weissia controversa Hedw.

Barbula australasiae (Hook. \& Grev.) Brid.

B. erinita (6) Schultz

B. torquata Tay1.

$B$. unguiculata Hedw.

BryoerythrophyzLum jamesonii (7) (Tayl.) Crum

Triquetrella papilzata (Hook.f. E Wils.) Broth.

Anomodon tasmanicus (8) Broth.

Encalyptaceae

Encalypta vulgaris Hedw.

Orthotrichaceae

Orthotrichum tasmanicum Hook.f. \& Wi1s.

Macromitrium archeri Mitt.

M. weymouthii Broth.

ULota Zutea (9) (Hook.f. E Wils.) Mitt.

Zygodon hookemi Hampe

Z. intermedius B.S.G.

Z. menziesii (Schwaegr.) Arnott

Funariaceae

Funaria apophysata (Tay1.) Broth.

F. glabra Tayl.

F. gracizis (Hook.f. \& Wils.) Broth.

F. hygrometrica Hedw.

F. producta (Mitt.) Broth.

Splachnaceae

Tayloria octobzepharis (Hook.) Mitt.

Bryaceae

Bryum argenteum Hedw.

$B$. bizzardieri Schwaegr.

B. blandum Hook.f. \& Wils.

B. capizzare Hedw.

B. crassum Hook. $f$. \& Wils.

$B$. dichotomw Hedw.

$B$. erythrocarpoides C. Mue11. E Hampe

$B$. Zaevigatum Hook.f. \& Wils.

B. micro-erythrocarpum C. Mue11. \& Kindb.

B. pachytheca C. Muell.

B. pseudotriquetrum (Hedw.) Gaertn., Meyer \& Scherb. Leptostomum inclinans $\mathrm{R} . \mathrm{Br}$.

Mielichnoferia bryoides (Harv.) Wijk \& Marg.
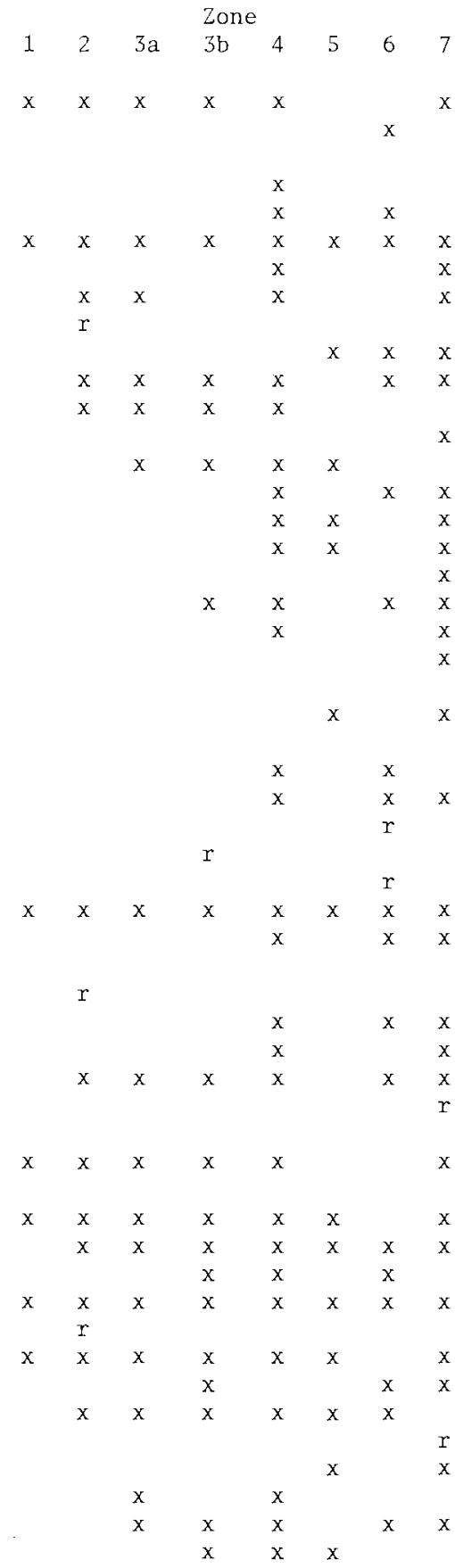
TABLE 2 (CONT.)

THE MOSSES OF THE MT WELLINGTON RANGE

Orthodontium lineare Schwaegr.

Pontia cruda (Hedw.) Lindb.

P. nutans (Hedw.) Lindb.

Aulacomniaceae

Leptotheca gaudichaudii Schwaegr.

Mitteniaceae

Mittenia plumuza (Mitt.) Lindb.

Rhizogoniaceae

Rhizogonium bifarium (Hook.) Schimp.

R. distichum (Sw.) Brid.

R. mioides (Hook.) Wils.

$R$. novae-holiandiae (Brid.) Brid.

Goniobryum subbasilare (Hook.) Lindb.

Hymenodon pilifer Hook.f. \& Wils.

Bartramiaceae

Bartramia hallerana Hedw.

$B$. cf. hampeana C. Muell.

B. papillata Hook.f. \& Wils.

Bartramidula pusilla (Hook.f. \& Wils.) Par.

Breutelia affinus (Hook.) Mitt.

B. elongata (Hook.f. E Wils.) Mitt.

B. pendula (Sm.) Mitt.

Conostomum pentastichum (Brid.) Lindb.

C. pusillum Hook.f. \& Wils.

Philonotis scabrifolia (Hook.f. E Wils.) Braithw. $P$. tenuis (Tayl.) Reichdt.

Hypnodendraceae

Hypnodendron comosum (Labi11.) Mitt.

H. spininervium (Hook.) Jaeg.

H. vitiense Mitt.

Rhacopilaceae

Rhacopilum convolutaceum (C. Mue11.) Reichdt.

Hedwigiaceae

Hedwigia ciliata (Hedw.) Ehrh.

H. integrifolia P. Beauv.

Rhacocarpus purpurascens (Brid.) Par.

Ptychomniaceae

Ptychomnion aciculare (Brid.) Mitt.

Glyphothecium sciuroides (Hook.) Hampe

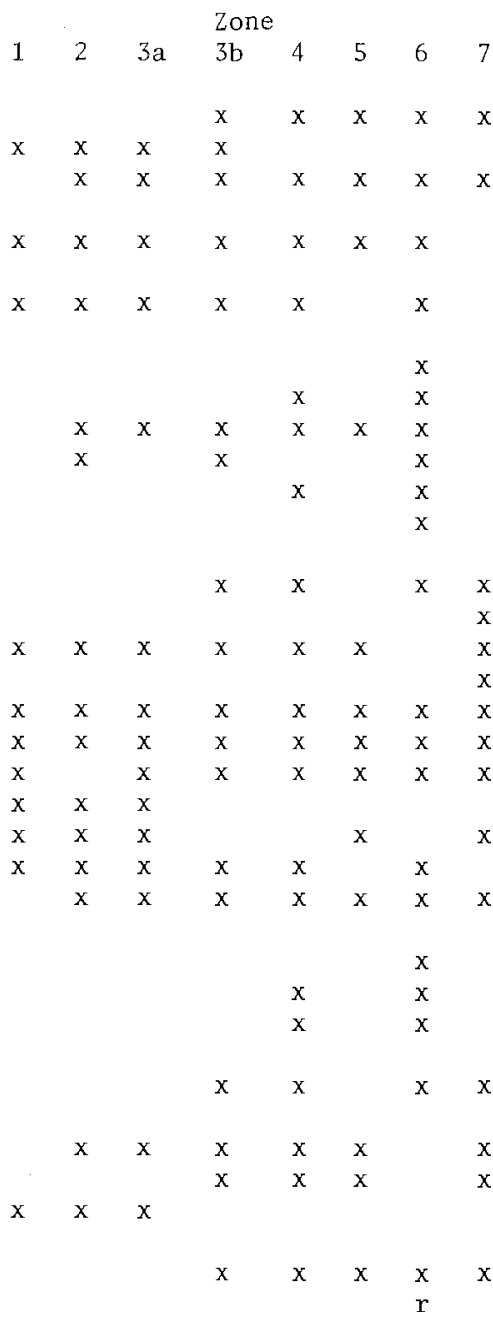

Lepyrodontaceae

Lepyrodon lagurus (Hook.) Mitt.

Meteoriaceae

Weymouthia cochlearifolia (Schwaegr.) Dix.

W. mollis (Hedw.) Broth.

Neckeraceae

Thamobryum pumizum (Hook.f. \& Wils.) Nieuwl.

Echinodiaceae

Echinodium hispidum (Hook.f. \& Wils.) Reichdt.

Lembophyl 1 aceae

Lembophyzlum divulsum (Hook.f. \& Wils.) Par.

Camptochaete arbuscula (Sm.) Reichdt.

C. Gracilis (Hook.f. \& Wils.) Par. 
TABLE 2 (CONT.)

THE MOSSES OF THE MT WELLINGTON RANGE

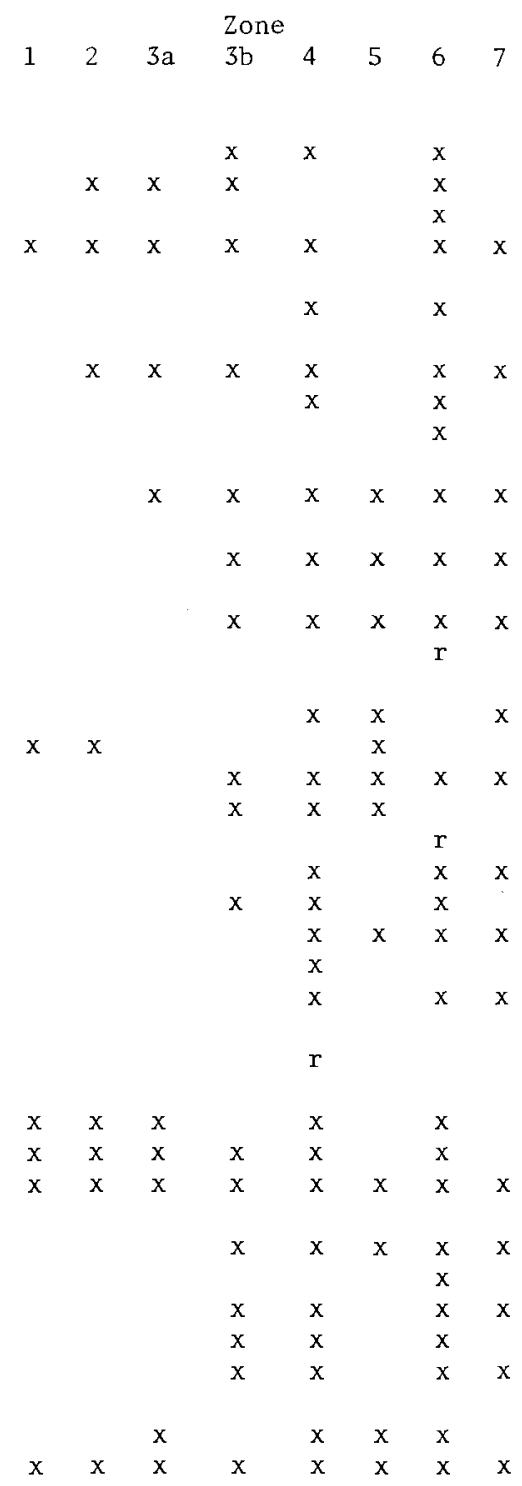

Footnotes (all references to Scott \& Stone 1976, are abbreviated S\&S).

(1) This may really be $S$. cymbifolioides C. Muell. (see S\&S p.453).

(2) Vitt (1980) showed that $A$. rupestris does not occur in the Southern Hemisphere and that material previously referred to by this name is mostly A. mutabizis.

(3) This species is not given as occurring in Tasmania by S\&S p.454.

(4) This species is not given as occurring in Tasmania by S\&S p.148, but we have also found it in Hartz Mtn National Park. 
TABLE 2 (CONT.)

THE MOSSES OF THE MT WELLINGTON RANGE

(5) Tortula rubra, found at Trestle Mtn, is a new Tasmanian record; we have also found it in Ben Lomond and Cradle Mountain National Parks.

(6) This name, as noted by S\&S p.454, replaces BarbuZa pseudopilifera C. Mue11. E Hampe.

(7) This species is listed in S\&S P.215 as B. binnsii (R.Br.ter.) Wijk \& Marg. (see Ratkowsky 1980b, p.367).

(8) This species was found at Knocklofty, Tolmans Hill, Browns River, Ridgeway Reservoir, etc. but sporophytes were not seen and its taxonomic position remains uncertain (see S\&్s p.218).

(9) According to Malta (1933), several species of ULota occur on Mt Wellington, which are supposedly easy to distinguish using vegetative characters.

(10) This name now replaces PterygophyZzum dentatum (Hook.f. \& Wils.) Dix. (see S\&gS p.454).

(11) Forms both with single and multiple cell papillae have been found. We believe the drawings in S\&S on p.403 and p.405, the latter purporting to be of T. Zaeviusculum (Mitt.) Jaeg., are of different forms of a sing1e species.

(12) This species, found near a creek behind the Cascade Brewery and also near Strickland Avenue, is a new record for Tasmania.

(13) The Mt Wellington material appears to be Rhynchostegium Zaxatum (Mitt.) Par. rather than $R$. tenuifolium (Hedw.) Reichdt., but as noted by S\&S p.431, a thorough revision of these two species is needed.

(14) The orange-to-yellow inflated alar cells seem to distinguish this species fron Hypnum cupressiforme Hedw.

of the species. For example, Allison and Child (1975), wherever it is cited, contains a brief description and a drawing of the relevant species.

The ordering of the families and of the five hepatic orders follows the proposal of Grolle (1972) with slight alterations, the taxonomic arrangement of the families within orders being alphabetic. Similarly, genera within families, and species within genera, are listed alphabetically. As with the mosses in table 2, presence of a species in a zone is indicated in table 3 by a " $x$ " or a " $r$ " (if it was found only once in the survey).

\section{DISCUSSION}

Rodway (1913-14, 17) 1isted a very high proportion of Tasmanian bryophytes as occurring on Mt Wellington, indicating the prominent place that this mountain has held in Tasmanian bryology. In our earlier survey of the vascular plants (Ratkowsky \& Ratkowsky 1976) it was found that more than one-third of the known flowering plants of Tasmania occurred on Mt Wellington. In the present census, 164 moss species and 130 liverwort species are reported, representing more than $60 \%$ of the approximately 270 known Tasmanian moss species and the approximately 210 known Tasmanian liverwort species. This reaffirms the importance of this mountain range as a significant natural reserve of representative Tasmanian vegetation.

This census has revealed several species that were previously unknown in Tasmania. Two moss species, Tortula rubra and Pseudoscleropodium purum, are new records for Tasmania, at least in the sense that they are not given by Scott and Stone (1976) as occurring in Tasmania. Cheilothela chilensis is also omitted from scott and Stone (1976), but we have also found it at Mt Field National Park. This distinctive plant, also known from South America, New Zealand, Victoria and New South Wales, but not common in any of those places, is now rather widespread as a colonizer of open ground at the higher elevations of Mt Wellington. No sporophytes have been found in Tasmanian material. 
TABLE 3

THE LIVERWORTS OF THE MT WELLINGTON RANGE

Order A: Anthocerotales

Anthocerotaceae

Megaceros sp. (1)

Phaeoceros sp. (2)

Order B: Marchantiales

Aytoniaceae

Astere Zla dmumondii (Tay1.) Schust. (3)

Lunulariaceae

LunuZaria cruciata (L.) Dum. (4)

Marchantiaceae

Marchantia berteroana Lehn. \& Lindenb. (5)

M. foliacea Mitt. (6)

Ricciaceae

Riccia sp. (7)

Order C: Metzgeriales

Aneuraceae

Aneura altemizoba (Hook.f. \& Tay1.) Tayl. (8)

A. rodwayi Hewson (9)

Riccardia aequiceltularis (Steph.) Hewson (10)

R. aZcicomis (Hook.f. \& Tayl.) Trev. (11)

R. cochleata (Hook.f. E Tayl.) Kuntze (12)

R. colensoi (Steph.) Martin (13)

R. crassa (Schwaegr.) Carringt, \& Pears. (14)

R. Zongiflora (Steph.) Hewson (15)

R. minima Carringt. G Pears. (16)

R. mpicola (Steph.) Hewson (17)

R. wattsiana (Steph.) Hewson (18)

Codoniaceae

Fossombronia pusizza (L.) Nees (19)

Hymenophytaceae

Hymenophyton flabelZatum (Labill.) Dum. ex Trev. (20)

Metzgeriaceae

M. atrichoneura spruce (21)

M. colensoi Steph. (22)

M. densiseta Steph. (23)

M. furcata (L.) Dum. (24)

M. Zeptoneura Spruce (25)

M. saccata Mitt. (26)

Pallaviciniaceae

PaZZavicinia xiphoides (Hook.f. \& Tay1.) Trev. (27)

Podomitrium phyzlanthus (Hook.) Mitt. (28)

Symphyogyna podophyzza (Thunb.) Mont. \& Nees (29)

Treubiaceae

Treubia Zacunosa (Col.) Prosk. (30)

T. tasmanica Schust. \& Scott (31)

Order D: Calobryales

Haplomitriaceae

Hap Zomitrium gibbsiae (Steph.) Schust. (32) $\begin{array}{llllllll}1 & 2 & 3 a & 3 b & 4 & 5 & 6 & 7\end{array}$

$\begin{array}{llll} & x & x & x\end{array}$

$\mathrm{x}$

$\begin{array}{lllllll}x & x & x & x & x & x & x \\ & & & x & & x\end{array}$

$r$

$\begin{array}{cccccccc} & \mathrm{x} & \mathrm{x} & \mathrm{x} & \mathrm{x} & & \mathrm{x} & \mathrm{x} \\ \mathrm{r} & & & & & & \\ \mathrm{x} & \mathrm{x} & \mathrm{x} & \mathrm{x} & \mathrm{x} & \mathrm{x} & \mathrm{x} & \mathrm{x} \\ & \mathrm{r} & & & & & & \\ & & & \mathrm{x} & \mathrm{x} & & \mathrm{x} & \\ \mathrm{x} & \mathrm{x} & \mathrm{x} & \mathrm{x} & \mathrm{x} & & \mathrm{x} & \\ & \mathrm{x} & \mathrm{x} & \mathrm{x} & \mathrm{x} & \mathrm{x} & \mathrm{x} & \mathrm{x} \\ & & & & & & \mathrm{x} & \\ & & \mathrm{x} & \mathrm{x} & & \mathrm{x} & \\ & & \mathrm{x} & \mathrm{x} & & \mathrm{x} & \\ & & & & & & \\ & & \mathrm{x} & \mathrm{x} & \mathrm{x} & & \mathrm{x} \\ & & & & & & \end{array}$

$\mathrm{x}$

r

$\mathrm{x}$

$\mathrm{x}-\mathrm{x} \quad \mathrm{x}-\mathrm{x}$

$\begin{array}{lll} & \mathrm{x} & \mathrm{x} \\ \mathrm{x} & \mathrm{x} & \mathrm{x} \\ & & \mathrm{r}\end{array}$

$\mathrm{r}$

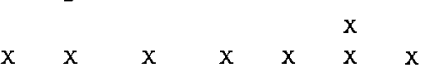

$r$ 
D.A. and A.V. Ratkowsky

TABLE 3 (CONT.)

THE LIVERWORTS OF THE MT WELLINGTON RANGE

Order E: Jungermanniales

Acrobolbaceae

Acrobolbus cinerascens

(Lehm. \& Lindenb.) Bast. (33)

Goebelobryum unguiculatum

(Hook.f. \& Tayl.) Grolle (34)

Lethocolea squamata (Tayl.) Hodgs. (35)

Tylimanthus pseudosaccatus Grolle (36)

T. tenelzus (Tayl. ex Lehm.) Mitt. (37)

Adelanthaceae

Adelanthus falcatus (Hook.) Mitt. (38)

Balantiopsaceae

Balantiopsis diplophyzla

(Hook.f. G Tayl.) Mitt. (39)

B. tumida Berggr. (40)

Isotachis intortifolia

(Hook.f. \& Tayl.) Gott. (41)

Neesioscyphus phoenicorhizus Grolle (42)

Cephaloziellaceae

Cephalozielza exiliflora (Tay1.) Steph. (43)

C. hirta (?) (Steph.) Schust. (44)

Chaetophyllopsidaceae

Chaetophyzlopsis whiteleggei

(Carringt. \& Pears.) Schust. (45)

Gymnomitriaceae

Gymnomitrion incompletum (Gott.) Schust. (46)

Herzogobryum aterrimum (Steph.) Grolle (47)

H. erosum (Carringt. \& Pears.) Grolle (48)

$H$. teres (Carringt. \& Pears.) Grolle (49)

Herbertaceae

Herbertus oldfieldianus (Steph.) Rodway (50)

Triandrophylium subtrifidum

(Hook.f. E Tayl.) Fulf. E Hatch. (51)

Jubul aceae

Frullania clavata (Hook.f. \& Tayl.) Tayl. (52)

F. falciloba (Hook.f. \& Tay1.) Lehm. (53)

$1 \quad 2 \quad 3 a \quad 3 b \quad 4 \quad 5 \quad 6 \quad 7$

F. monocera (Hook.f. \& Tayl.) Tayl. (54)

F. probosciphora Tayl. (55)

F. rostrata (Hook.f. \& Tay1.) Hook.f. \& Tayl. (56)

F. scandens Mont. (57)

Jungermanniaceae

Anastrophylzum schismoides (Mont.) Steph. (58)

Andrewsianthus perigonialis

(Hook.f. \& Tay1.) Schust. (59)

Cryptochiza grandiflora

(Lindenb. \& Gott.) Grolle (60)

Cuspidatuza monodon (Hook.f. G Tay1.) Steph. (61)

Jamesoniella colorata (Lehm.) Schiffn. (62)

J. tasmanica (Hook.f. \& Tayl.) Steph. (63)

Jungermannia inundata Hook.f. \& Tayl. (64)

$J$. orbiculata (Col.) Grolle (65)

Lophozia tasmanica Schust. (66)

$\mathrm{x} \quad \mathrm{x}$

$\mathrm{x}$

$\mathrm{x} \quad \mathrm{x}$

$\mathrm{x}$

$x$

$\mathrm{x} \times \mathrm{x}$

$\begin{array}{llllll} & x & x & x & x & x\end{array}$

$x \quad x \quad x$

$\begin{array}{lllllll}x & x & x & x & x & x & x\end{array}$

$\begin{array}{llllllll}x & x & x & x & x & x & x & x\end{array}$

$\begin{array}{lll}\mathrm{x} & \mathrm{x} & \\ \mathrm{x} & & \\ \mathrm{x} & \mathrm{x} & \\ \mathrm{x} & \mathrm{x} & \mathrm{x} \\ & & \\ \mathrm{x} & \mathrm{x} & \mathrm{x} \\ \mathrm{x} & \mathrm{x} & \mathrm{x}\end{array}$


TABLE 3 (CONT.)

THE LIVERWORTS OF THE MT WELLINGTON RANGE

Lejeuneaceat

Cheilo lejeunea albo-virens

(Hook.f. E Tayl.) Hodgs. (67)

C. mimosa (Hook.f. \& Tay1.) Schust. (68)

Diplasiolejeunea plicatizoba

(Hook.f. G Tayl.) Grolle (69)

Harpalejeunea Latitans

(Hook.f. \& Tayl.) Grolle (70)

Lejeunea dmumondii Tayl. (71)

Lepicolea cea

Lepico lea scolopendra

(Hook, ) Dun. ex Trev. (72)

\section{Lepidolaenaceae}

Gackstroemia weindorferi (Herz.) Grolle (73)

Lepido Zaena brachyclada

(Tay1, ex Lehm.) Trev. (74)

L. reticulata (Hook.f. \& Tayl.) Trev. (75) Lepidoziaceae

Acromastigum colensoanum (Mitt.) Evans (76)

Bazzania adnexa (Lehm. \& Lindenb.) Trev. (77)

B. monizinervis (Lehm. \& Lindenb.) Trev. (78)

Hygrolembidium acrocladum (Berggr.) Schust. (79)

H. custrale (Steph.) Grolle (80)

Kurzia hippuroides (Hook.f. \& Tayl.) Grolle (81)

K. tenax (Grev.) Grolle (82)

Lepidozia gZaucophyzza (Hook.f. \& Tayl.) Tayl. (83)

L. Zaevifolia (Hook.f. \& Tayl.) Tayl. (84)

L. pendulina (Hook.) Lindenb. (85)

L. uZothrix (Schwaegr.) Lindenb. (86)

Telaranea centipes (Tayl.) Schust. (87)

T. gottscheana (Lindenb.) Hodgs. (88)

Zoopsis argentea (Hook.f. \& Tayl.) Hook.f. (89)

Z. Leitgebiana (Carringt. \& Pears.) Bast. (90)

Lophocoleaceae

Chiloscyphus aliodontus

(Hook.f. \& Tayl.) Hodgs. (91)

C. bizzardieri (Schwaegr.) Nees (92)

C. coalitus (Hook.) Nees (93)

C. echineZzus (Lindenb. \& Gott.) Mitt. (94)

C. fissistipus (Hook.f. \& Tayl.) Tayl. (95)

C. kirkii steph. (96)

C. limosus Carringt. \& Pears. (97)

C. triacanthus (Hook.f. \& Tayl.) Steph. (98)

C. tridentatus Mitt. (99)

CZasmatocolea marginata (Steph.) Grolle (100)

C. strongyZophyZZa (Hook.f. \& Tayl.) Grolle (101) Hepatos tolonophora paucistipula

(Rodw.) Engel (102)

H. rotata (Hook.f. \& Tayl.) Engel (103)

Leptophyzzopsis Zamus (Mitt.) Schust. (104)

Leptoscyphus sp. (105)

Lophocolea bidentata (L.) Dun. (106)

L. excipulata Steph. (107)

L. gunniana Nees (108) 
TABLE 3 (CONT.)

THE LIVERWORTS OF THE MT WELLINGTON RANGE

L. Zenta (Hook.f. \& Tayl.) Tayl. (109)

L. Zeucophylla (Hook.f. \& Tayl.) Tayl. (110)

L. muricata (Lehm.) Nees (111)

L. semiteres (Lehm.) Mitt. (112)

P1 agiochil aceae

Plagiochila baileyana Steph. (113)

$P$. circinatis

(Lehm. \& Lindenb.) Lehm. \& Lindenb. (114)

$P$. fasciculata Lindenb. (115)

$P$. incurvicolza

(Hook.f. \& Tayl.) Hook.f. \& Tayl. (116)

P. pZeurata

(Hook.f. \& Tayl.) Hook.f. \& Tayl. (117)

P. radiculosa Mitt. (118)

$P$. ratkowskiana $\mathrm{H}$. Inoue (119)

P. retrospectans (Nees) Nees (120)

P. strombifolia (Tayl.) Hook.f. \& Tay1. (121)

Pseudolepicoleaceae

Isophyllaria attenuata (Rodw.) Hodgs. (122)

Termoma townrowii Schust. (123)

Radulaceae

Raduza buccinifera

(Hook.f. \& Tay1.) Tayl. (124)

Raduza sp. (125)

Scapaniaceae

DipZophyZZum domesticum (Gott.) Steph. (126)

Schistochilaceae

Schistochila ciliata (Mitt.) Steph. (127)

S. Zehmanniana

(Lindenb.) Carringt. \& Pears. (128)

Trichocoleaceae

Trichocolea mollissima

(Hook.f. \& Tay1.) Gott. (129)

T. rigida Schust. (130)

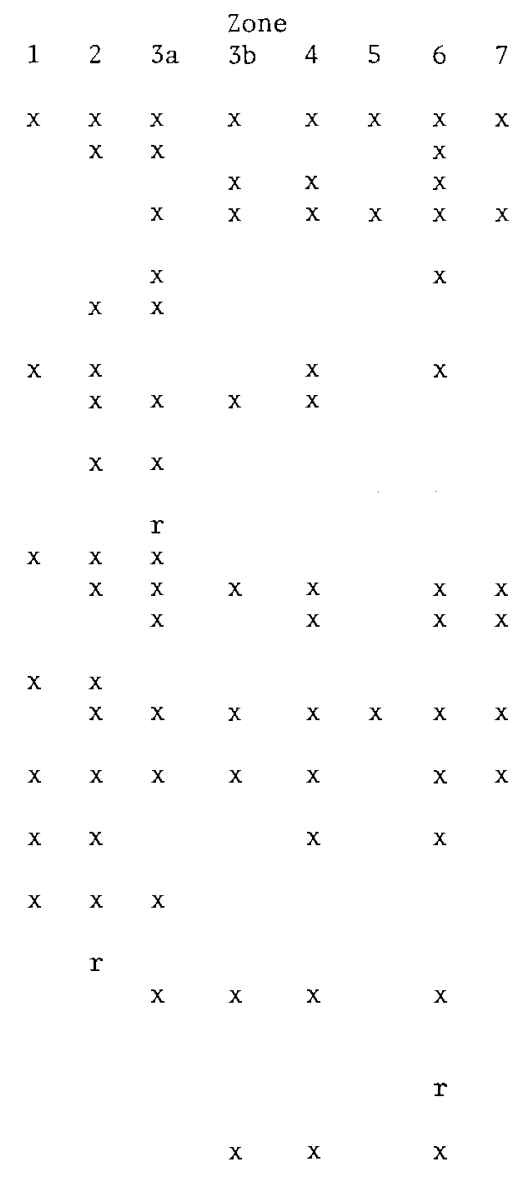

Footnotes

(1) Carrington \& Pearson 1888, pp.11-12; Bastow 1888, p.283, plates XXXV and XLIII, both as Anthoceros Zongispirus. Spores greenish, minutely verruculose; elators with a single, spiral band.

(2) Bastow 1888, p.283, plate XXXV as Anthoceros Zaevis; A1lison \& Child 1975, pp.283-284. Spores yellowish, papillose; elators rudimentary, lacking spira1 bands.

(3) Bastow 1883, p.282; Rodway 1917, pp.57-58, both as Fimbriaria drummondii.

(4) Campbe11 1965a, pp.31-42, figs.1-21; Al1ison \& Child 1975, pp.271-273.

(5) Campbe11 1965b, pp.122-126, figs.1-10; A1lison \& Child 1975, pp.272-277.

(6) Campbel1 1965b, pp.126-130, figs.11-24; Al1ison \& Child 1975, pp.274-278.

(7) The Australian species of the genus Riccia have recently been revised by Na-Thalang 1980. Tasmanian material was not studied, however.

(8) Hewson 1970a, pp.186-188, figs.1,3-4.

(9) Hewson 1970a, pp.188-189, figs.1,4; Allison \& Child 1975, pp.252-253.

(10) Hewson 1970b, pp.79-80, fig.6.

(11) Hewson 1970 b, p.77, fig.6.

(12) Hewson 1970b, pp.89-91, fig.8; Al1ison \& Child 1975, pp.254-255. 
THE LIVERWORTS OF THE MT WELLINGTON RANGE

(13) Hewson 1970b, pp.103-104, fig.11.

(14) Hewson 1970b, pp.104-106, fig.11.

(15) Hewson 1970b, pp.88-89, fig.8.

(16) Hewson 1970b, p.84, fig. 7.

(17) Hewson 1970b, pp.99-100, fig. 10.

(18) Hewson 1970b, pp.91-92, fig.9.

(19) Allison \& Child 1975, pp.233-234. The genus is a very difficult one and is badly in need of revision; some of the Mt Wellington material may be other species of Fossombronia.

(20) Campbel1 et al. 1975, pp.593-600, fig.3; Allison \& Child 1975, pp.245-246.

(21) Hodgson 1961, pp.720-721, figs.13-18; Kuwahara 1966, pp.235-236, figs.12f and $22 \mathrm{a}$.

(22) Hodgson 1961, pp.723-724, figs.23-24; Kuwahara 1966, p.224, fig.19a; Allison \& Child 1975, pp.263-264.

(23) Kuwahara 1960, p.22, fig.8; Kuwahara 1966, p.236.

(24) Hodgson 1961, pp.714-717, figs.1-5; Kuwahara 1966, pp.236-237, fig.11b; Allison \& Child 1975, pp.262-263.

(25) Hodgson 1961, pp.717-718, figs.6-9; Kuwahara 1966, pp.223-224, fig.12b; Allison \& Child 1975, pp.261-262, all as M. hamata Lindb., an illegitimate name according to Enge1 (1976).

(26) Hodgson 1961, pp.721-723, figs.19-22; Kuwahara 1966, pp.215-216, fig.16, as Austrometzgeria saccata.

(27) Hllsse1 de Menendez 1961a, pp.268-270, figs.3-4; Allison \& Child 1975, p.237.

(28) Bastow 1888, p.269, plate XXXI; A11ison \& Child 1975, pp.246-247.

(29) Hässel de Menendez 1961b, pp.255-259, figs.9-10; Campbe11 et a. 1975, pp.593-600, fig.2; Allison \& Chi1d 1975, pp.238-241, a11 as S. hrymenophyzlum.

(30) Schuster \& Scott 1969, pp.233-238, figs.1-2, 4-7; Al1ison \& Child 1975, pp. $232-233$.

(31) Schuster \& Scott 1969, pp.248-250, figs.1-2, 4-5.

(32) Campbe11 1959, pp.245-254, figs.1-33, as Calobryum gibbsiae; Schuster 1967, pp.21-28, figs.IX-X; Hässe1 de Menendez 1970, pp.235-238, fig.1.

(33) Bastow 1888, p.242, p1ate XVI; Hodgson 1958, pp.581-582, fig.7.

(34) Grolle 1962, pp.135-144, figs.1-4.

(35) Bastow 1888, p.243, plate XVII, as $L$. drummondii; Hodgson 1958, p.582; Allison \& Child 1975, pp.191-192.

(36) Bastow 1888, p.241, p1ate XVI; Grolle 1963a, pp.391-394, fig.121.

(37) Bastow 1888, p.241, plate XVI; Hodgson 1958, pp.573-575, fig.2; Allison $\&$ Child 1975, pp.185-187.

(38) Bastow 1888, p.235, p1ate XII; Allison \& Chi1d 1975, pp.193-194.

(39) Bastow 1888, p.243, p1ate XVII; Engel 1968, pp.109-112, figs.326-464.

(40) Enge 1 1968, pp.106-108, figs.276-325.

(41) Hodgson 1949a, pp.29-30; Hatcher 1960, pp.602-604, figs.483-558. We are herein treating Isotachis grandis Carringt. \& Pears. as a likely synonym.

(42) Grolle 1964a, pp.27-29, fig.4. Previous1y known only from the type in New Zea1and.

(43) Schuster 1972, pp.190-193; Allison \& Child 1975, pp.195-196.

(44) Schuster 1972, pp.190-212. Differs from C. exiliflora in that the cells are armed with spinous teeth; plants green, not becoming reddish. A thorough revision of this genus is needed.

(45) Hodgson \& Al1ison 1962, pp.152-154, figs.35-39, as Anoplostomim whiteleggei; Schuster 1974, pp.172-174, fig.1.

(46) Schuster 1966a, pp.279-280.

(47) Grolle 1980b, pp.326-327; Grolle 1975a, pp.71-74, fig.l, as $H$. filarium.

(48) Carrington \& Pearson 1888, pp.8-9, plate XLI, as Cesia erosa; Grolle 1966a, pp. 231-234, fig. 4 .

(49) Carrington \& Pearson 1888, pp.9-10, plate XLII, as Jungermannia teres; Grolle 1966a, pp.222-226, fig.2. 
TABLE 3 (CONT.)

THE LIVERWORTS OF THE MT WELLINGTON RANGE

(50) Bastow 1888, pp.248-249, plate XXI, as Sendtnera juniperina. Similar to, but generally considered to be specifically distinct from $H$. alpinus (Steph.) Hodgs., illustrated in Allison \& Child 1975, pp.37-38.

(51) Fulford \& Hatcher 1961, pp.348-351; Allison \& Child 1975, pp.38-40.

(52) Hattori 1979a, pp.328-329, fig.4.

(53) Hattori 1979a, pp.336-337, fig.10; also pp.340-342, fig.13, and pp.361-362, fig. 27 as $F$. forsythiana and $F$. wattsiana respectively.

(54) Hattori 1979a, pp.347-349, fig.18; also Hattori 1979b, pp.132-134, fig.36 as $F$. hampecana.

(55) Hattori 1979b, p.139; also pp.131-132, fig.35, as F. falsa.

(56) Bastow 1888, pp.265-266, plates XXIX and XXXVI, as FruZZania diplota; Hattori 1979a, pp.352-353, fig.21.

(57) F. depZanata Mitt. and F. subdep Zanata Steph. are probably conspecific as both have flattened perianths lacking ventral keels; Hodgson 1949b, pp.380-381, figs.19-20 as F. subdeplanata and F. deplanata; Allison \& Child 1975, pp.217-218, as $F$. dep Zanata.

(58) Allison \& Child 1975, pp.93-94.

(59) Schuster 1966a, pp.280-281.

(60) Grolle 1971, pp.19-22, fig.4; Al1ison \& Child 1975, pp.98-101.

(61) A1lison \& Child 1975, pp.94-95.

(62) Grolle 1971, pp.81-85, fig.22; Allison \& Child 1975, pp.102-103.

(63) Grolle 1971, pp.55-59, fig.15; Allison \& Child 1975, pp.101-102.

(64) Ván̆a 1975, pp.299-303, figs.17-19; Allison \& Child 1975, pp.97-98.

(65) Váña 1975, pp.303-306, figs.19-21; Possibly just an aquatic form of Jungermannia inundata.

(66) Schuster 1968, pp.484-485; Schuster \& Engel 1975, p.469.

(67) Schuster 1963, p.245.

(68) Schuster 1963, p.245.

(69) Grolle 1975b, pp.75-79, fig.1; Allison \& Child 1975, pp.228-229, both as Diplasiozejeunea Zyratifolia.

(70) Grolle 1980a, pp.239-243, fig.2.

(71) Bastow 1888, pp.258-259, plate XXVII, as Lejeunea guniana.

(72) Scott 1960 , pp.152-154, figs.60-95.

(73) Grolle 1967, pp.20-24, figs.5-6; Allison \& Child 1975, pp.54-55.

(74) Grolle 1967, pp.43-46, figs.13-14.

(75) Grolle 1967, pp.46-47, fig.13.

(76) Hodgson 1954, pp.21-22, fig.14.

(77) Hodgson 1954, pp.13-14, figs.2 and 7; Allison \& Child 1975, pp.82-85. B. involuta (Mont.) Trev. is possibly another form of this highly variable species.

(78) Hodgson 1954, pp.10-11, fig.5.

(79) Herzog 1951, pp.488-491, figs.6-7, as Lembidium isodictyon; Grolle 1966b, p.229.

(80) Herzog 1951, pp.491-494, figs.8-9, as Lembidium stereophyZlum; Grolle 1966b, pp. $229-230$.

(81) Hodgson 1956, p.615, as Lepidozia hippuroides; Carrington \& Pearson 1888, pp.2-3, p1ate XXXVII, as Lepidozia capiZlaris var. geniculata; Kurzia compacta (Steph.) Grolle is treated herein as probably conspecific; cf. Hodgson 1956, p.616, fig.27, as Lepidozia compacta.

(82) Grolle 1963b, p.174; Gro11e 1964b, p.80.

(83) Hodgson 1956, pp.594-596, fig.3; Allison \& Child 1975, pp.64-65.

(84) Hodgson 1956, p.597, fig.5; Allison \& Child 1975, p.66.

(85) Hodgson 1956, pp.601-602, fig.10; Allison \& Child 1975, pp.71-72.

(86) Hodgson 1956, pp.593-594, fig.2; Allison \& Chi1d 1975, pp.63-64.

(87) Hodgson 1956, pp.605-606, fig.16, as Lepidozia centipes; Allison \& Child 1975, pp. $76-77$.

(88) Hodgson 1956, pp.609-611, fig.20, as Lepidozia gottscheana; A11ison \& Child 1975, pp. $77-78$. 
(89) Scott 1969, pp.165-168, figs.2-3; A1lison \& Child 1975, pp.90-91.

(90) Scott 1969, p.169, figs.1, 6-7; A11ison \& Chi1d 1975, pp.90-91.

(91) Hodgson 1943, p.42, fig. 12 .

(92) Hodgson 1943, pp.36-37, fig.25; Al1ison \& Child 1975, pp.144-145.

(93) Hodgson 1943, pp.41-42, fig.8; A11ison \& Chi1d 1975, pp.146-149.

(94) Hodgson 1943, pp.45-46, fig. 10.

(95) Hodgson 1943, pp.48-49, fig.13; Allison \& Child 1975, pp.150-151.

(96) Rodway 1917, p.102.

(97) Bastow 1888 , p. 239, plates XV \& XXXIX.

(98) Hodgson 1943, pp.47-48, fig.20; A11ison \& Chi1d 1975, pp.147, 149-150.

(99) Bastow 1888, p.239, plate XIV; Hodgson 1943, Pp.46-47, fig. 14.

(100) Bastow 1888, p.233, plate XII, as Jungermannia marginata.

(101) Hodgson 1953, pp.338-339, fig.10, as Lophocolea strongyzophyzza.

(102) Engel 1979, pp.103-107, fig.7

(103) Enge1 1979, pp.98-103, fig.5.

(104) Schuster 1963, pp.269-270; Hodgson 1943, p.45, fig.7, as Chizoscyphus Zaxus.

(105) Bastow 1888 , p. 225, plate VI, as Leioscyphus chizoscyphioides. The relationship of this species to Leptoscyphus exponsus (Lehm.) Grolle and Leptoscyphus australis (Hook.f. \& Tayl.) Schust. needs study.

(106) Hodgson 1953, pp.354-355, fig.32; Allison \& Child 1975, pp.140-141; see footnote 109.

(107) Rodway 1917, p.91.

(108) Hodgson 1953, pp.337-338, fig.11, as $L$. notophyzza.

(109) Hodgson 1953, pp.350-351, fig.27; Al1ison \& Child 1975, pp.136-137. Extraordinarily variable vegetative1y; possib1y $L$. subporosa Mitt., L. decurrens Herz., and even $L$. bidentata (L.) Dum., together with this, are all forms of a single species.

(110) Hodgson 1953, pp.343-344, fig.19; A11ison \& Chi1d 1975, pp.132-133.

(111) Hodgson 1953, pp.342-343, fig.18; Allison \& Child 1975, pp.131-132.

(112) Hodgson 1953, pp.334-335, fig.8, as L. heterophyzzoides; Allison \& Child 1975, pp. $130-131$.

(113) Inoue \& Schuster 1971, pp.173-180, figs.61-63.

(114) Inoue \& Schuster 1971, pp.123-130, figs.44-45.

(115) Inoue \& Schuster 1971, pp.180-187, figs.64-66.

(116) Inoue \& Schuster 1971, pp.77-82, figs.26-27.

(117) Inoue \& Schuster 1971, pp.110-115, figs.40-41.

(118) Inoue \& Schuster 1971, pp.117-121, figs.42-43.

(119) Inoue 1980, pp.141-144, figs.1-10. This new species, described from a collection on Mt Wedge, is widespread on many Tasmanian mountains.

(120) Inoue \& Schuster 1971, Pp.203-213, figs.72-74.

(121) Inoue \& Schuster 1971, pp.131-138, figs.46-48.

(122) Hodgson 1972, p.111; Hodgson 1967, fig.5; Schuster 1966b, pp.69-73, fig.1, the latter two as $I$. murrayana.

(123) Schuster $1966 \mathrm{c}$, pp.351-355, figs.48-50.

(124) Cast1e 1967, pp.70-74, fig.34; A11ison \& Child 1975, pp.201-202.

(125) This is probably a group of species characterized by an inflated 1obule and by cells that tend to be somewhat mamillose, including $R$. compacta Castle 1963, pp.43-45, fig.20, R. tasmanica Steph. (Cast1e 1963, pp.36-38, fig.17), R. tabularis Steph. (Cast1e 1963, pp.38-41, fig.18), R. oneurysmalis (Hook.f. \& Tay1.) Gott. (Castle 1963, pp.28-30, fig.12) and R. wattsiana Steph. (Castle 1963, pp.45-47, fig. 21).

(126) Allison \& Child 1975, pp.104-105.

(127) Hodgson 1941, p.188, fig.31; Schuster 1971, fig.8; A11ison \& Child 1975, pp. 116, 121-122.

(128) Hodgson 1941, pp. 184-185, figs.10-11.

(129) Schuster 1968, pp.445-447, plate 50, figs.8-11; Ratkowsky 1980a, pp.79-80.

(130) Schuster 1968, pp.447-449, plate 50, figs.1-7; Ratkowsky 1980a, p.80. 
Amongst the liverworts, several new Tasmanian records have been found in the survey area. Perhaps the most remarkable is Neesioscuphus phoenicorhizus, a very distinctive plant, of which we have material from at least six locations in the survey area. The species was previously known only from the type collection near Arthurs Pass in New Zealand [Grolle 1964a], and as all other known species of this genus grow in tropical localities, the taxonomic affinities of this species have not been established with certainty. Chaetophyllopsis whiteleggei, Haplomitrium gibbsiae and Hygrolembidium australe are also new to Tasmania, these records having been obtained in this survey. In addition, two other species, also obtained in this survey but not listed in table 3 , were identified tentatively by Dr. R. Grolle (pers.comm.) as belonging to the genera Cephazozia (Dum.) Dum. and Paracromastigum Fulf. \& J. Tayl. respectively. These could, upon further study and subsequent specific identification, also prove to be new Tasmanian records.

TABLE 4

NUMBER OF SPECIES IN EACH VEGETATION ZONE

\begin{tabular}{|c|c|c|c|c|c|c|c|c|}
\hline & \multicolumn{8}{|c|}{ Zone } \\
\hline & 1 & 2 & $3 a$ & $3 b$ & 4 & 5 & 6 & 7 \\
\hline Mosses & 43 & 62 & 62 & 78 & 108 & 61 & 102 & 82 \\
\hline Liverworts & 34 & 70 & 63 & 50 & 69 & 25 & 67 & 29 \\
\hline Total bryophyte count & 77 & 132 & 125 & 128 & 177 & 86 & 169 & 111 \\
\hline
\end{tabular}

Table 4 ists the number of species in each vegetation zone, obtained by counting the entries in the columns of table 2 and table 3 . Mosses have their maximum occurrence in the zones of middle and lower elevation, the greatest numbers being in Zones 4 , 6 , 7 and $3 b$. Liverworts occur most frequently in Zone 2, followed by Zones 4 , 6 and $3 a$, with comparatively low numbers in the low elevation dry sclerophyll environments of $Z$ zone 7. Ogden and Powell (1979), from a study of woody plants at Mt Field National Park, Tasmania, concluded that species richness increases with altitude to reach a maximum in the subalpine zone. This conclusion does not hold for Mt Wellington, as a tally of woody species from the survey by Ratkowsky and Ratkowsky (1976) shows that the greatest abundance occurs in Zone 4 and Zone 7 , the numbers declining distinctly in the higher elevation zones. The numbers in table 4 enable one to examine the effect of elevation on bryophyte richness. The greatest moss richness is seen to occur in the middle and lower zones, whereas liverworts are spread out over a wide range of altitudes, although they are relatively scarce in zones 5 and 7 .

One may examine the question further by grouping closely related zones and counting the number of species that occur exclusively in one zone group. The basis for grouping the zones was taken from Ratkowsky and Ratkowsky (1977), where several methods of hierarchical cluster analysis were used. It was found that Zones 3b, 4 and 6 clustered to form a gully/wet sclerophyl1 forest group; Zones 1,2 and 3 formed a high elevation group of treeless or woodland habitats; Zone 7 formed a dry sclerophyll forest group of its own; and zone 5 had no close neighbour and could not be grouped with other zones. Table 5 lists the number of species restricted to one of the following three zone groups, with Zone 5 omitted: any or all of Zones 1, 2 and $3 a$; any or all of Zones $3 \mathrm{~b}, 4$ and 6 ; exclusive to Zone 7 .

The results in table 5 show that the mosses occur predominantly in the gully/wet sclerophyll forest group, with much smaller numbers of species occurring either in the high elevation group or dry sclerophyll. The liverworts, however, exhibit a different pattern, with half the species in table 5 occurring in the high elevation zone group. An almost equally large number of liverwort species occurs in the gully/wet sclerophyll zone group, with only three species being exclusive to dry sclerophyll forest. The 
NUMBER OF SPECIES RESTRICTED TO CERTAIN ZONE GROUPINGS (omitting zone 5 from consideration)

Species occurring only in zones 1,2 and/or 3 a
Species occurring only in Zones $3 b, 4$ and/or 6
Species occurring only in Zone 7
Mosses

Liverworts
16
42
13

3

results in table 4 and table 5 indicate that mosses predominate in the middle zones (gullies and wet sclerophyll communities) but are also relatively abundant in the lower elevations (dry sclerophyll), whereas liverworts are abundant both at middle and higher elevations but are relatively infrequent in the dry sclerophyll forests.

Only a few species previously known to have occurred on Mt Wellington, from citations in Rodway (1913-14, 17) or from specimens preserved in the Tasmanian Herbarium, have not been found in the present survey. The largest and most striking of these is Neckera pennata Hedw., a pendent species previously known from a few east-facing gullies. The current absence of this species from Mt Wellington may be traced to alienation of environment as a result of land clearance and house building, or may be due to the devastating bushfire of 7 February 1967. Although there has clearly been a strong recovery of the bryophyte flora since the fire, as evidenced by the large number of species found in this survey, epiphytic species are slow to recover, and it may be expected that, barring further fires, various epiphytic moss and liverwort species presently missing may be found on Mt Wellington in the future.

\section{ACKNOWLEDGEMENTS}

Various bryologists have been most helpful in providing identifications, with particular thanks going to Dr. Riclef Grolle of Jena, without whose willing assistance the list of liverwort species would have been far less complete. Other identifications were provided by Drs. E.0. Campbel1, J.J. Enge1, S. Hattori, H.J. Hewson, H. Inoue, G.A.M. Scott, R.D. Seppelt, K. Yamada and Y. Kuwahara. Not all of the identifications provided by the above-mentioned people have necessarily been uncritically accepted, and one of us (D.A.R.) assumes responsibility for any mistakes in nomenclature that appear in tables 2 and 3 . We thank Dr. A.E. Orchard, Curator of the Tasmanian Herbarium (Ho), for making preserved material of Tasmanian mosses and liverworts available for study.

\section{REFERENCES}

Allison, K.W. and Child, J., 1975: THE LIVERWORTS OF NEW ZEALAND. University of Otago Press, Dunedin.

Bastow, R.A., 1886-1887: Tasmanian mosses. Pap. Proc. R. Soc. Tasm. (1885): 318-320, 337-341, 395-399; Pap. Proc. R. Soc. Tasm. (1886): 38-102.

1888: Tasmanian hepaticae. Pap. Proc. R. Soc. Tasm. (1887): 209-289.

Campbell, E.O., 1959: The struture and development of Calobryum gibbsiae steph. Trans. R. Soc. N.Z., 87: 245-254.

1965a: Lunularia in New Zealand. Tuatara, 13: 31-42.

1965b: Marchantia species of New Zealand. Tuatara, 13: 122-136.

, Markham, K.R. and Porter, L.J., 1975: Dendroid liverworts of the order Metzgeriales in New Zealand. N.Z. J. Bot., 13: 593-600.

Carrington, B. and Pearson, W.H., 1888: Description of new or rare Tasmanian hepaticae. Pap. Proc. R. Soc. Tasm. (1887): 1-20.

Castle, H., 1963: A revision of the genus Radula. Part II. Subgenus Acroradula. Section 6. Saccatae. Rev. Bryol. Lichénol., 32: 1-48. 
Castle, H., 1967: A revision of the genus Radula. Part II. Subgenus Acroradula. Section 1l. Complanatae. Rev. Bryol. Lichenol., 35: 1-94.

Engel, J.J., 1958: A taxonomic monograph of the genus Balantiopsis (Hepaticae). Nova Heduigia, 16: 83-130.

, 1976: Metzgeria hamata Lindb., an illegitimate name of Hepaticae. Lindbergia, 3: 219-220.

1979: Austral Hepaticae X. A revision of HepatostoZonophora Engel et Schust., nom. nov. (Hepaticae). J. Hattori Bot. Lab., 46: 91-108.

Fulford, M. and Hatcher, R.E., 1961: The genus Triandrophyzlum - some nomenclatural changes. Bryologist, $64: 348-351$.

Grolle, R., 1962: Goebelobryum, eine neue marsupiale Lebermoosgattung. J. Hattori Bot. Lab. , 25: 135-144.

1953a: Ein neuer Tylimanthus aus Tasmanien. Nova Hedwigia, 6: 391-394. 1963b: Über Kurzia v. Martens. Rev. Bryol. Lichénol., 32: 166-180. 1964a: Neesioscyphus, eine neue Lebermoosgattung mit gedrehten

Sporogonklappen. Österr. bot. Zeitschrift, 111: 19-36.

1964b: Notulae hepaticologicae. xv. Neue Notizen über Kurzia v. Mart. und

verwandte. J. Jap. Bot., 39: 79-81.

, 1966a: Herzogobryum, eine beblätterte Lebermoosgattung mit dorsal

verzahnten Merophyten. Österr. bot. Zeitschrift, 113: 221-234.

, 1966b: Miscellanea hepaticologica (61-70). J. Jap. Bot., 41: 225-232. 1967: Monographie der Lepidolaenaceae. J. Hattori Bot. Lab. , 30: 1-53. 1971: Jomesoniezla und verwandte. Feddes Rep., 82: 1-99.

1972: Die Namen der Familien und Unterfamilien der Lebermoose

(Hepaticopsida). J. Bryol., 7: 201-236.

1975a: Ein neues Herzogobryum aus Tasmanien und N.S.Wales: H. filarizm sp. nov. Feddes Rep., 86: 71-74.

, 1975b: Diplasiolejeunea in Australasien. Feddes Rep. , 86: 75-82.

1980a: Über Harpalejeunea in Australasien. J. Hattori Bot. Lab. , 47: 237-244.

1980b: Miscellanea hepaticologica 201-210. J. Bmyol., 11: 325-334.

Hässel de Menéndez, G.G., 196la: Las especies sudamericanas del género Pallavicinia. Boletin de la Sociedad Argentina de Botánica, 9: 261-282.

196lb: Las especies argentinas del género Symphyogyna. Boletin de la Sociedad Argentina de Botánica, 9: 233-260. , 1970: El género Haplomitrium (Hepaticae). Revista del Museo argentino de ciencias naturales "Bemardino Rivadavia", 3: 235-238.

Hatcher, R.E., 1960: A monograph of the genus Isotachis (Hepaticae). Nova Hedwigia, $2: 573-508$.

Hattori, S., 1979a: A revision of the Australasian species of the genus Frmizania, Hepaticae, I. J. Hattori Bot. Lab. , 45: 323-363.

1979b: A revision of the Australasian species of the genus Frullania, Hepaticae, II. J. Hattori Bot. Lab., 46: 119-153.

Herzog, Th., 1951: Revision der Lebermoosgattung Lembidium Mitt. Apkiv f. Bot., Series 2, 1: 471-503.

Hewson, H.J., 1970a: The family Aneuraceae in Australia and New Guinea. I. The genus Aneura. Proc. Linn. Soc. N.S.W., 94: 173-193.

, 1970b: The family Aneuraceae in Australia and New Guinea. II. The genus Riccardia. Proc. Linn. Soc. N.S.W., 95: 60-121.

Hodgson, E.A., 1941: Review of the New Zealand species of Schistochila, with notes on Colenso's species. Trans. R. Soc. N.Z., 71: 181-194.

, 1943: A review of the New Zealand species of the genus Chiloscyphus.

Trans. R. Soc. N.Z., 73: 27-52.

, 1949a: A review of the New Zealand species of the genus Isotachis. Rev. Bryol. Lichénol., 18: 25-31.

- 1949b: A review of the New Zealand species of the genus Fruzlania. Trans. R. SOC. N.Z., 77: 361-389.

1953: A review of the New Zealand species of the genus Lophocolea with notes on Chiloscyphus. Trans. R. Soc. N.Z., 80:329-358. 
Hodgson, E.A.: 1954: A review of the New Zealand species of the genera Bazzania and Acronastigum. Trans. R. Soc. N.Z., 82: 7-24.

1956: A reyiew of the New Zealand species of the genus Lepidozia. Trans. R. SOC. N.Z., 83: 589-620.

, 1958: Marsupial genera of New Zealand. Trans. R. Soc. N.Z., 85: 565-584. 1961: A review of the New Zealand species of the genus Metzgeria. Trans. R. SOC. N.Z., 88: 713-725.

, 1967: A miscellany of taxonomic notes, Part 2. Trans. R. Soc. N.Z., Botany, 3: 175-198.

$109-118$ 1972: A miscellany of taxonomic notes, Part 3. J. R. Soc. N.Z., 2:

and Allison, K.W., 1962: A review of the New Zealand species of the genus Temnoma and of Anoplostoma, a new genus. Trans. R. Soc. N.Z., Botany, 1: $139-154$.

Inoue, H., 1980: A new species of Plagiochiza (Hepaticae) from Tasmania. Brunonia, 3: 141-144.

and Schuster, R.M., 1971: A monograph of the New Zealand and Tasmanian Plagiochilaceae. J. Hattori Bot. Lab. , 34: 1-225.

Kuwahara, Y., 1960: The genus Metzgeria in Pacific Oceania. J. Hattori Bot. Lab. , 23: $3-28$.

1966: the family Metzgeriaceae in North and South East Asia, Pacific Oceania, Australia and New Zealand. Rev. Bryol. Lichénol., 34: 191-239.

Malta, N., 1932: A survey of the Australasian species of Ulota. Acta Horti bot. Univ. Zatv. (Raksti), 7: 1-24.

Na-Thalang, Obshant, 1980: A revision of the genus Riccia (Hepaticae) in Australia. Brunonia, 3: 61-140.

Ogden, J. and Powell, J.A., 1979: A quantitative description of the forest vegetation on an altitudinal gradient in the Mount Field National Park, Tasmania, and a discussion on its history and dynamics. Aust. J. Ecol., 4: 293-325.

Ratkowsky, D.A., 1980a: Tasmanian and New Zealand Trichocoled (Hepaticae). Bryologist, $83: 78-82$.

, 1980b: Some further synonyms of Bryoerythrophyzzum jomesonii (Tayl.) Crum. Bryologist, 83: 367 .

and Ratkowsky, A.V., 1976: Changes in the abundance of the vascular plants of the Mount Wellington Range, Tasmania, following a severe fire. Pap. Proc. R. Soc. Tasm., 110: 63-90.

1977: Plant communities of the Mt Wellington Range, Tasmania. Aust. J. Ecol., 2: 435-445.

Rodway, L., 1913-14: Tasmanian Bryophyta. I. Mosses. Pap. Proc. R. Soc. Tasm. (1912): 3-24, 87-138; (1913): 177-263. (Issued separately in 1914 as a single publication, $163 \mathrm{pp}$. , Royal Soc. Tasm., Hobart).

1917: Tasmanian Bryophyta. II. Hepatics. Pap. Proc. R. Soc. Tasm. (1916): 51-143. (Issued separately in 1916 as a single publication, 93 pp., Royal Soc. Tasm. , Hobart).

Sainsbury, G.0.K., 1953-1956: Notes on Tasmanian mosses from Rodway's Herbarium. 1. Pap. Proc. R. Soc. Tasm., 87: 83-91; 2. ibid. 89: 1-11; 3. ibid. 89: 13-20; 4. ibid. 89: 21-35; 5. ibid. 89: 37-43; 6. ibid. 89: 45-53; 7. ibid. 90: 35-39; 8. ibid. $90: 41-47$.

Schuster, R.M., 1963: Studies on antipodal Hepaticae. I. Annotated keys to the genera of antipodal Hepaticae with special reference to New Zealand and Tasmania. J. Hattori Bot. Lab., 26: 185-309.

, 1966a: Studies in Lophoziaceae. Rev. Bryol. Lichénol., 34: 240-287.

$59-136$.

- 1966c: A memoir on the family Blepharostomataceae, II. Candolzea, 21:

$241-355$.

- 1967: Studies on Hepaticae. XV. Calobryales. Nova Heduigia, 13: 1-76.

, 1968: Studies on Hepaticae. XXIX-XLIV. A miscellany of new taxa and new range extensions. Nova Heduigia, 15: 437-529.

, 1971: Studies of antipodal Schistochilaceae and scapaniaceae. Bulz. Nat. Sei. Mus. Tokyo, 14: 609-660. 
Schuster, R.M., 1972: Studies on Cephaloziellaceae. Nova Hedwigia, 22: 121-265. 1974: Studies on antipodal Hepaticae. XI. The Chaetophyllopsidaceae: their taxonomy, phylogeny and phytogeographic affinities. Bull. Nat. Sei. Mus. Tokyo, 17: 163-180.

and Engel, J.J., 1975: Austral Hepaticae, IV. Notes on Lophozia subgenus Protolophozia Schust., with diagnosis of a new South American species. J. Bryol., $8=465-474$.

and Scott, G.A.M., 1969: A study of the family Treubiaceae (Hepaticae; Metzgeriales). J. Hattori Bot. Lab., 32: 219-268.

Scott, E.B., 1960: A monograph of the genus Lepicolea. Nova Hedurgia, 2: 129-172.

Scctt, G.A.M., 1969: The New Zealand species of Zoopsis (Hepaticae). Rec. Dom. Mus., Wellington, 6: 159-174.

and Stone, I.G., (with illustrations by C. Rosser), 1976: THE MOSSES OF SOUTHERN AUSTRALIA, ACademic Press, London.

Ván̆a, J., 1975: Studien über die Jungermannioideae (Hepaticae). 8. Jungermannia subg. Plectocolea und subg. Solenostoma in Australien, Neuseeland und Ozeanien. Folia Geobot. Phytotax., Praha, 10: 277-323.

Vitt, D.H., 1980: A comparative study of Andreaea acutifolia, A. mutabilis and A. Pupestris. N.Z. J. Bot., 18: 367-377. 